EVS25

Shenzhen, China, Nov 5-9, 2010

\title{
Total Solutions of System Integration and Development Technology for $\mathrm{EV}$
}

\author{
Chien-An Chen ${ }^{1}$, Ming-Chih Lin ${ }^{2}$, and Cheng-Shun Jiang ${ }^{3}$ \\ ${ }^{1,2,3}$ Electronic Vehicle and System Verification Group R \& D Division, Automotive Research and Testing Center, \\ Changhua, 50544, Taiwan \\ E-mail: chienan@artc.org.tw
}

\begin{abstract}
In recent years, the research of Battery Electric Vehicle (BEV) focuses on energy storage systems, drive system and control strategy. However, one of the important subjects to enhance the performance of BEV is to research the parameters of transmission until the power battery and other technologies are mellow. For the BEV drive system parameters design, such as motor power and output torque as well as transmission gear ratio and the matching have significant impacts on each other for driving range and performance of $\mathrm{BEV}$. Hence, in BEV research and developmental phase, in order to meet the requirements of vehicle performance access to the best structure, generally it must base on different needs to optimize the parameters of the drive system and to consider the combination of vehicle operation among the various components. For this reason, it will be conducive to the rapid development and be widely used for BEV. Therefore, in this paper, the discussion of the design, analysis, matching and system integration of BEV component parameters is proposed, which can be used as a reference for the future development of BEV.
\end{abstract}

Keywords: Battery Electric Vehicle (BEV), System Integration, Parameter Matching.

\section{Induction}

In recent years, due to the awareness of environmental and oil depletion issues, governments and manufacturers around the world all put into the electric vehicle research and popularization. The U.S. government allocates 2.4 billion U.S. dollars to subsidize the research and development for the new electric vehicles, batteries and components. Its goal of electric vehicle development project is to reach 1 million electric vehicles in 2015. Meanwhile, German plans to have 1 million electric vehicles (PEV, PHEV) in 2020. In United Kingdom, the Plugged-In Places program will take 3 million pounds to support the building of charging device to meet growing future demand for electric vehicle driving. In some Asian countries, China and Japan have also put into vehicle and component research and charging station layout plans. From above, all show that the electric vehicle research work is urgent.

The topics of Electric Vehicle have been very popular and the overall performance of electric vehicle (speed, acceleration time, climbing ability, endurance ... etc.) are often concerned. However, in recent years, the security protection, fault diagnosis, failure mode-related issues are also concerned gradually. On functional performance, it mainly depends on the correct matching of components while security 
protection, fault diagnosis, failure mode, condition analysis and detection depend on the vehicle planning and design. On the other hand, power management systems and vehicle network communication technology are also popular for current research topics. In this paper, it uses Mitsubishi Colt-Plus model to build a pure electric vehicle that integrate key technologies of Taiwan companies and combine with the Automotive Research \& Testing Center (ARTC) deployment and development of the vehicle communication network and energy management system technology to achieve an integrating and developing platform for $\mathrm{EV}$.

System integration of BEV is a complex matter involving several disciplines. The disciplines include mechanical design, control logic analysis, power electronics and thermal management. Overall, the major challenges concern the integration between the battery and the electric propulsion system. However, how to communicate and integrate various systems will be the major theme of development of electric vehicles. Therefore, communication between the each system will be a new research issue on the development of systems integration.

The structure of this paper is as follows. Firstly, the structure, operation principle and key technology of the BEV are introduced in Section 2. In Section 3, matching and design of propulsion driving system for BEV is analyzed, it includes vehicle parameter, performance requirement, matching and design of the $\mathrm{BEV}$ component, assembly and layout of the BEV and performance estimation and simulation of the BEV. The complete performance of electric vehicles and other main features of the integrated deployment technology are discussed in Section 4. Finally, our conclusions are presented in Section 5 .

\section{Structure of the BEV}

\subsection{Structure and Operation Principle of the BEV}

The power unit, chassis, body parts and electrical device are the mainly established elements of the BEV. Among them, the power unit and control system is the kernel of BEV and that is the biggest difference between the internal combustion engine vehicle and BEV. The power unit is composed of the drive motor, power supply and motor speed control device and other components. In recent years, owing to the electric vehicles dedicated chassis have not yet development completed. Hence, in the body of electric vehicles and electrical device are the same as conventional cars, besides, it joined with the auxiliary control system to enhance handling and ride comfort of the auxiliary system (electric power steering system, electric air-conditioning system ...), its main structure is shown in Figure 1.

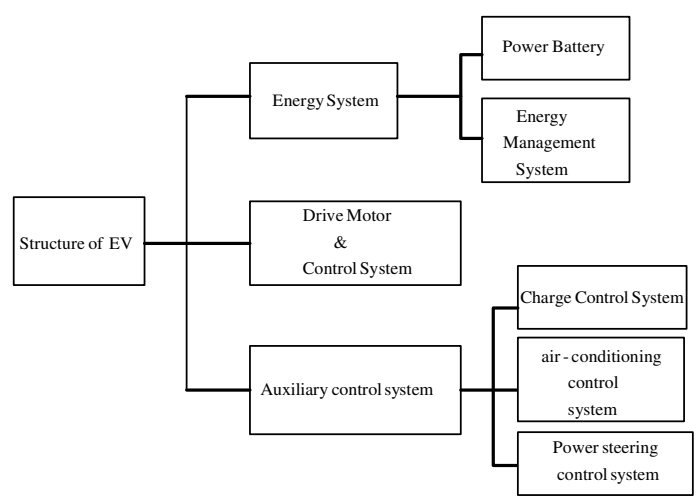

Figure 1: The main structure of the BEV

Base the structure of electric vehicle, which main operator axiom is depicted in Figure 2. First, its dominance is via the EMS allocation power battery power to power systems (subsystems). Secondly, according to the acceleration or braking command signal, and then by the main control system to drive motor, finally drive the wheels through the transmission mechanism to rotate.

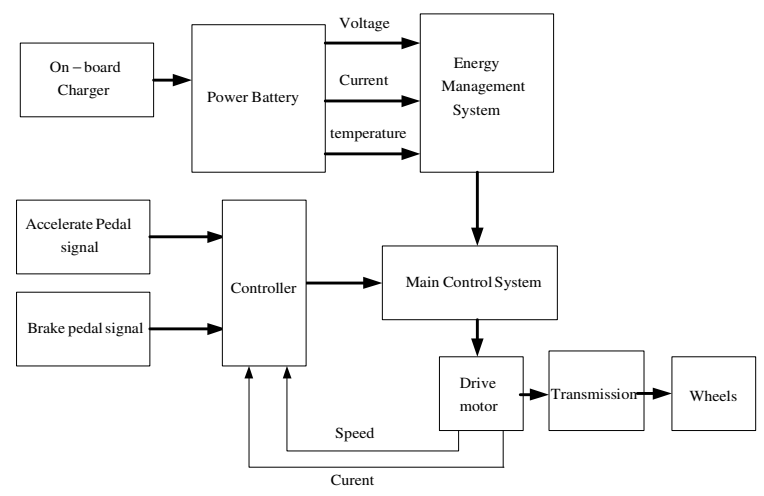

Figure 2: The main operator axiom of BEV

\subsection{Key Technology of the BEV}

The key technologies of developing pure electric vehicles are electric drive, control technology battery technology, the entire vehicle technology battery management technology and energy management technology will each be analyzed as follows:

Electric vehicle drive system is composed of drive motor, control system (including motor drives, controllers), transmission, and wheels posed. Control system is responsible for the integration of accelerator pedal, brake, stop, forward, reverse, neutral, steering wheel, and other signals. Through the control unit by the signal processing, followed by 
input to the motor drive, and then control the drive motor speed and torque, and by transmission device and then drive the wheels. As the drive motor usage is a key component to get the good performances of electric vehicle. Hence, the drive motor should have the major requirements as follows:

(1) Have wide speed range

(2) a larger starting torque

(3) Small size, light weight, and high efficiency

(4) Reliability

(5) High Constant Power Speed Ratio (CPSR)

(6) High Overload Capability

Power Battery technology is a key factor to develop BEV successfully due to it is the energy source of the $\mathrm{BEV}$ and its quality will affect vehicle performance. Due to the energy density, power density, and life cycle are the main adoption index for power battery. Hence, the major requirements of BEV currently are as follows:

(1) High energy density $(\mathrm{w} \bullet \mathrm{h} / \mathrm{kg})$ and power density

(W/ kg)

(2) Long life cycle

(3) High $\mathrm{C}$ rate charge and discharge

(4) Low cost

(5) Low internal resistance and low self-discharge rate

(7) To obey regulations of the working temperature range for vehicle

(8) Safety of usage

(9) Consistent properties

A well designed electric vehicles, which in addition to good mechanical efficiency, electrical drive performance, an appropriate energy source. Besides, it should also have a system, which can monitor and manage all of the electric power systems. The system is composed of the battery management system (BMS) and energy management system (EMS). BMS main function is to monitor the battery voltage, current and temperature indicator, discharge status, and discharge depth, for avoiding overcharge, overdischarge and battery voltage unbalance between the components and control the temperature of the battery. EMS preside over several missions, such as collecting each subsystem operation data of vehicle to conduct monitoring and diagnosis, to provide charging control, and to display of residual energy etc. EMS monitoring system is the main data acquisition part, and the control unit is mainly based on information provided by the monitoring system to control various devices in order to achieve optimal energy management.

Electric vehicle is a comprehensive high-tech product, besides batteries and motor, the car body itself contains a lot of technology. Therefore, vehicle technology should be considered as follows.

(A) light-weight materials such as magnesium, Aluminum, high-quality steel and composite materials, optimization of the structure of the automobile itself can be reduced by $30 \%$ to $50 \%$;

(B) To achieve braking and downhill the energy Regeneration

(C) Ability of low rolling resistance tires to reduce energy consumption

(D) Streamlined vehicle body design, this allows vehicles reduce air resistance

So, it will be able to reduce overall energy consumption, to improve performance.

\section{Matching and Design of Propulsion Driving System for BEV}

Propulsion driving system is the kernel of electric vehicle. The electric vehicle propulsion driving system parameters, (such as motor power, transmission gear ratio, etc.), are except significant for vehicle dynamic performance and cost. Hence, in order to meet the performance requirements, in this proposed paper will focus on the choice of $\mathrm{BEV}$ propulsion driving system parameters and the matching analysis.

\subsection{Parameters and performance requirements}

In this proposed research, we choice a medium-sized sedan to be a development platform, the vehicle parameters (Dimensions) are shown in Table 1.

Reference to the specification and plan to the performance indicators of the target EV is shown as follows.

(1)Maximum speed $\geq 130 \mathrm{~km} \mathrm{/} \mathrm{h;}$ (2)Cruise range $\geq 80 \mathrm{~km}$ @ $60 \mathrm{~km} \mathrm{/} \mathrm{h;}$ (3)Maximum climbing capability $\geq 30 \%$;

(4) Acceleration time $\leq 10 \mathrm{sec}$ from $0 \mathrm{~km} / \mathrm{h}$ to $60 \mathrm{~km} \mathrm{/h}$.

Table 1 The parameters of BEV

\begin{tabular}{|l|l|}
\hline \multicolumn{1}{|c|}{ Parameters } & \multicolumn{1}{c|}{ Value } \\
\hline Overall Length & $4185 \mathrm{~mm}$ \\
\hline Overall Width & $1680 \mathrm{~mm}$ \\
\hline Overall Height & $1550 \mathrm{~mm}$ \\
\hline Curb weight & $1200 \mathrm{~kg}$ \\
\hline Tires & P185/55R15 \\
\hline Rolling resistance coefficient & 0.015 \\
\hline Aerodynamic resistance coefficient & 0.328 \\
\hline
\end{tabular}

3.2 Matching and Design of the BEV Component 
Due to the motor, drive controller, gearbox and battery are the main components of electric vehicle propulsion driving system and in order to meet the design requirements, so the required specifications will be design and matched [1]-[4].

The gear ratio selection of transmission will be based on the vehicle's dynamic performance requirements, so the choice of gear ratio should meet the expectations of the maximum speed, maximum climbing capability and acceleration time.

The gear ratio of transmission for EV has two ways, one is single speed fixed gear another is multigearshift. If the selected range of motor speed is wide enough, achieve the capability of power performance and the highest speed requirements. That can use single speed fixed gear to reduce the weight, volume and cost of electric vehicles. The selected technique is as follows.

(1) The upper limit of gear ratio: The upper limit of gear ratio is calculated by the peak speed of motor $\left(n_{m}\right)$ and the maximum speed of vehicle $\left(v_{m}\right)$.

$$
i \leq \frac{0.377 n_{m} r}{v_{m}} .
$$

where $r$ is the wheel radius.

(2) The lower limit of gear ratio : The lower limit of gear ratio is calculated by the following two ways to determine the maximum transmission ratio.

(a)The peak speed of motor $\left(n_{m}\right)$ corresponds to the output torque $\left(T_{n m}\right)$ and maximum speed of vehicle $\left(v_{m}\right)$ corresponds to the driving resistance $\left(F_{n m}\right)$ determine the lower limit of gear ratio.

$$
i \geq \frac{F_{n m} r}{\eta_{t} T_{n m}}
$$

where

$\eta_{t}$ : Transmission of efficiency

$$
\begin{aligned}
F_{n m} & =m g f_{r}+\frac{C_{d} A}{21.15} v_{m}^{2} . \\
T_{n m} & =9549 \frac{P_{e}}{n_{m}} .
\end{aligned}
$$

(b) The lower limit of gear ratio is decided by the maximum output torque of the motor $\left(T_{a m}\right)$ and the maximum climbing angle $\left(\alpha_{m}\right)$ corresponds to the total running resistance $\left(F_{a m}\right)$.

$$
i \geq \frac{F_{a m} r}{\eta_{t} T_{a m}} .
$$

where

$$
\begin{aligned}
& v_{a}: \text { speed of maximum climbing. } \\
& F_{a m}=m g\left(f_{r} \cos \alpha_{m}+\sin \alpha_{m}\right)+\frac{C_{d} A}{21.15} v_{a}^{2}
\end{aligned}
$$

In addition to meet the basic running resistance, vehicle has to provide loading capability of climbing and acceleration. Therefore, the motor in electric vehicle is not only to meet the requirements of rated power, but also to meet the maximum overloading torque and instantaneous power requirements. According to above requirements, the electrical parameters of design skills is discuss as follows.

(1) Based on the maximum speed electric vehicle choice:

In order to ensure the electric vehicles to achieve the maximum speed on flat road, so the rated power should not be chosen less than the power of total running resistance. Therefore select the conditions is described as follows.

$$
P_{e} \geq \frac{1}{\eta_{t}}\left(\frac{m g f_{r}}{3600} v_{m}+\frac{C_{d} A}{76140} v_{m}^{3}\right) .
$$

where

$m$ :curb weight ;

$f_{r}:$ Rolling resistance coefficient ;

$C_{d}$ : Aerodynamic resistance coefficient ;

A : Frontal area ;

$\eta_{t}:$ Transmission efficiency.

(2) Choose the motor peak power by according to the power consumption of the maximum loading.

In this part, that is to estimate the required acceleration or climbing maximum instantaneous power, its selection criteria is described as follows.

$$
\begin{aligned}
P_{m} & \geq \frac{1}{\eta_{t}}\left(\frac{m g f_{r} \cos \alpha}{3600} v_{\alpha}+\frac{m g \sin \alpha}{3600} v_{\alpha}\right. \\
& \left.+\frac{C_{d} A}{76140} v_{\alpha}^{3}\right) .
\end{aligned}
$$

(Operating point and the climbing speed non- corresponding the motor rated speed)

$$
P_{m} \geq \frac{1}{\eta_{t}}\left(\frac{T_{a m} \cdot n_{m}}{9549}\right)
$$

(Necessary power under the corresponding motor rated speed)

The total energy of battery is decided by the driving range of the electric vehicles, hence the total energy limits of battery can be obtain from to design the driving range.

$$
E_{b}=L \cdot e .
$$

where

$E_{b}:$ The capacity of power battery, kwh; 
$L$ : Cruise range, $\mathrm{km}$;

$e$ : Energy consumption per unit distance , Kwh/Km:

According to above discussion, we combine with the Advisor 2002 and Matlab to development the analysis programs as shown in figure 3 , it can analysis the matching and design of the BEV component.

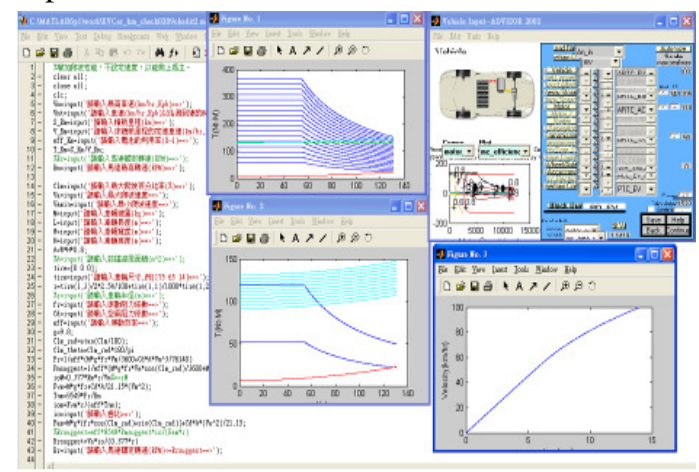

Figure 3: The analysis programs of the matching and design techniques

\subsection{Assembly and Layout of the BEV}

Considering the space of the vehicle, we remove the components, it including engine, transmission, water tank, fuel tank, exhaust pipes, etc. Then, we decided the motor, gear box, inverter, brake vacuum pump and automotive air-conditioning system (AAC) to be installed in the engine room, and all the batteries installed in the trunk. Finally, completed the configuration of the engine room is shown in Figure 4 and Figure 5.

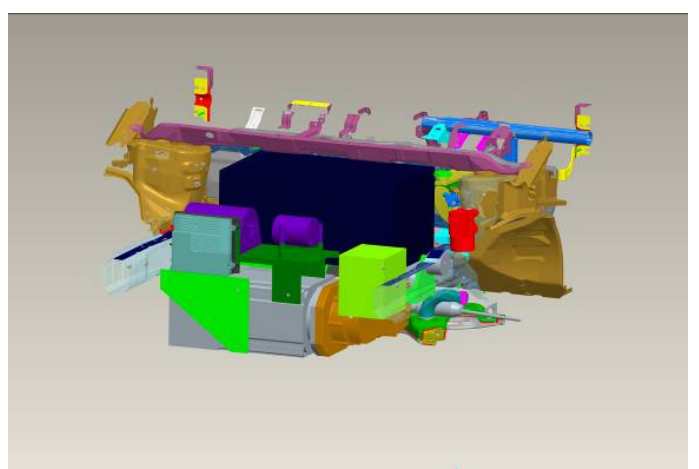

Figure 4: The configuration of the engine room

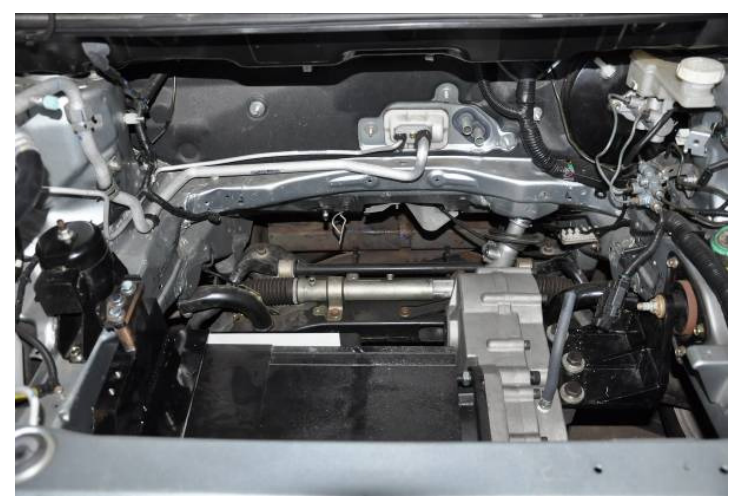

(a)

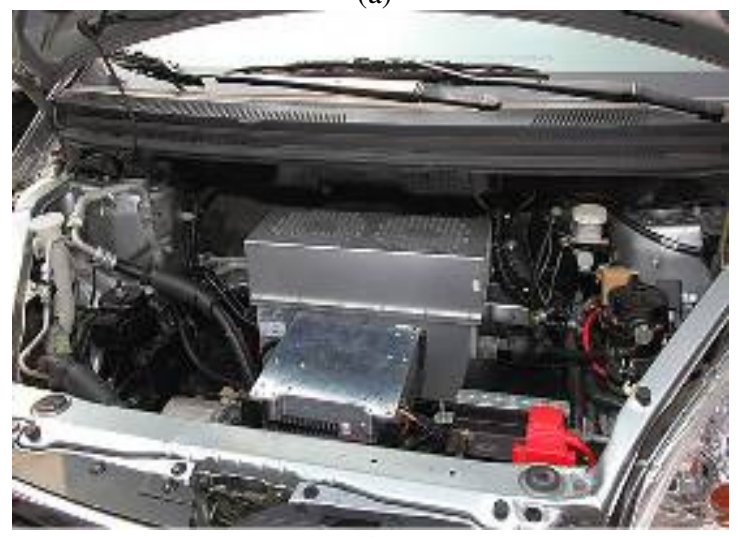

(b)

Figure 5: Propulsion Driving System in engine room

\subsection{Performance Estimate and Simulation of the BEV}

In order to accelerate the vehicle component specifications match schedule, it is written the program which is according to the section 3.2. In the figure 6 , the relationship map of the motor T-N curve and the running resistance is depicted. From the figure 6 , we choose the motor rated power is $30 \mathrm{~kW}$ and the peak power is $69 \mathrm{~kW}$, then consider the actual motor characteristic curve and estimate out the accelerate time. The $0-100 \mathrm{~km} / \mathrm{hr}$ acceleration time, motor $\mathrm{P}-\mathrm{N}$ and $\mathrm{T}-\mathrm{N}$ graph is shown in figure 7 . Finally, the overall performance estimate results of the proposed BEV in this paper are shown in Table 2 . 

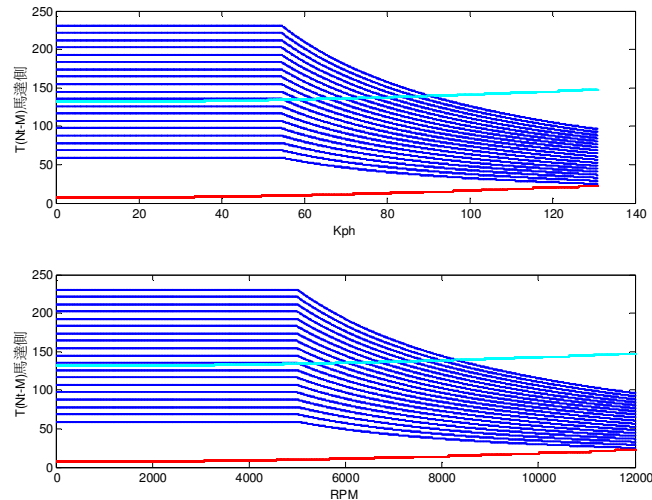

Figure 6: The relationship map of the motor T-N curve and the running resistance
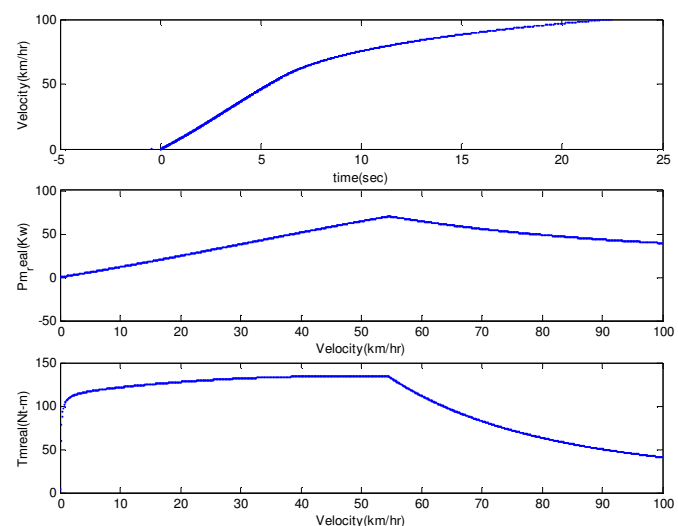

Figure 7: The $0-100 \mathrm{~km} / \mathrm{hr}$ acceleration time, motor P-N and T-N

Table 2 the overall performance estimate results of the proposed BEV

\begin{tabular}{|l|l|}
\hline \multicolumn{1}{|c|}{ Performance } & \multicolumn{1}{c|}{ Value } \\
\hline Max. Speed & $130.9 \mathrm{kph}$ \\
\hline Acceleration time (0 60kph) & $6.0651 \mathrm{sec}$ \\
\hline Maximum climbing capability & $30.314 \%$ \\
\hline
\end{tabular}

\section{System Integration for BEV}

In the BEV, it includes Energy Management System (EMS), Battery Management System (BMS), Propulsion Management System (PMS)...etc. However, how to communicate and integrate various systems will be the major theme of development of electric vehicles [5]-[7]. In addition to BMS, EMS and PMS, the system integration of BEV in this proposed paper also including automotive airconditioning system (AAC), electric power steering system and electric braking system. We reached the goal of communication, control and diagnostics with vehicle network (CAN BUS). The structure of EV system integration is shown in Figure 8.

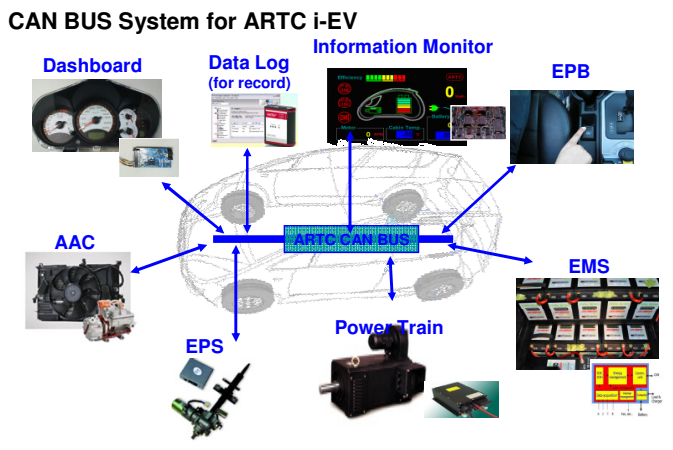

Figure 8: The structure of EV system integration.

\section{Verifying Results and Conclusion}

Through the above analysis and discussion, the electric vehicle in this paper is finished by proposed design flow. It has been proved through several tests, which is shown as follows.

- Power Performance Test

- Maximum Speed, acceleration ( Figure 9)

- Climbing Test

- Climbing performance test

-Emission \& Fuel Economy Testing Lab to LDV

( Figure 10)

- Cruise Range Test with FTP75 and Cruise (60Km / h)

The electric vehicle overall performance specifications are shown in table 2.

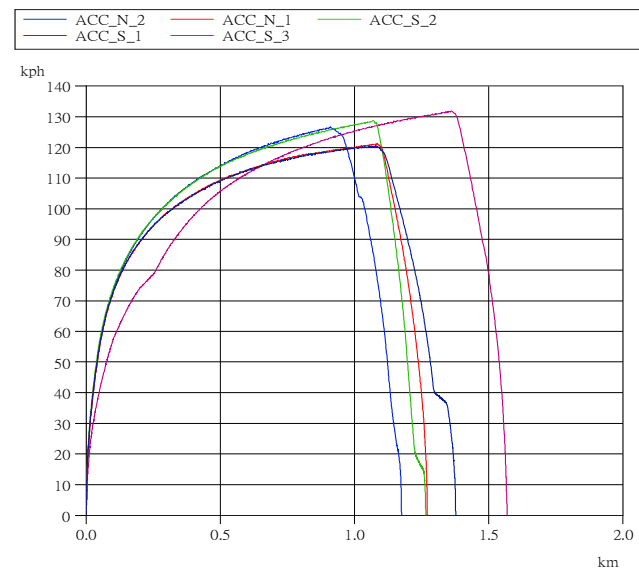

Figure 9: The results of the acceleration test. 


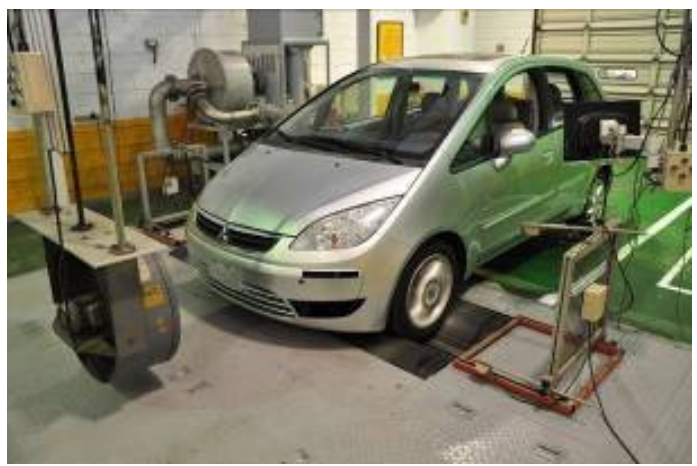

Figure 10: Emission \& Fuel Economy Testing Lab to LDV.

Table 2 The electric vehicle overall performance specifications

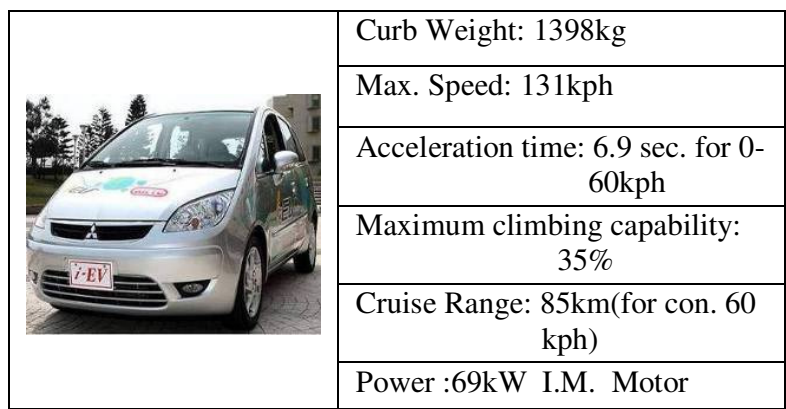

In this paper, the total solutions of system integration and development technology for EV are presented. The analysis, design, matching, layout and integration technology are employed to $\mathrm{EV}$ development to get better performance. Therefore, the system integration and development technology can be easily applied to BEV. Finally, we employ the results of verification to validate the proposed design formula.

\section{References}

[1] L. Wang and X. Wen, Dynamic Match and Optimizing Design of Electric Vehicle Powertrain, 1999 IEEE International Vehicle Electronics Conference (IVEC 1999), 1999, 387-390.

[2] X. Zeng, H. Min, X. Xu and Q. Wang, Parameter Design for Power Train and Performance Simulation of Electrical City Bus, 2008 IEEE Vehicle Power and Propulsion Conference (VPPC 2008), 2008, 1-5.

[3] W. Wang, Q. Wang, X. Zeng, X. Yang and H.Min, Parameter Matching of Induction Motor for Electric Vehicle Based on Orthogonal Design, 2009 IEEE International Conference on Mechatronics and Automation (ICMA 2009), 2009, 1455-1459.

[4] H. Zhao, B. Zhang and B. Zhang, Research on Parameters Matching of Parallel Hybrid Electric Vehicle Powertrain, 2008 IEEE Vehicle Power and Propulsion Conference (VPPC 2008), 2008, 1-4.

[5] H. Yoo, S. K. Sul, Y. Park and J. Jeong, System Integration and Power Flow Management System Integration and Power Flow Management Supercapacitors and Batteries, 2007 Applied Power Electronics Conference (APEC 2007), 1032-1037.
[6] H. Yoo, S. K. Sul, Y. Park and J. Jeong, System Integration and Power Flow Management System Integration and Power Flow Management Supercapacitors and Batteries, IEEE Transactions on Industry Applications, Vol. 44, 2008, pp: 108-114.

[7] C. Quigley, ELECTRONIC SYSTEM INTEGRATION FOR HYBRID AND ELECTRIC VEHICLES, Hybrid Vehicle Conference, 2006, pp: 125-140.

\section{Authors}

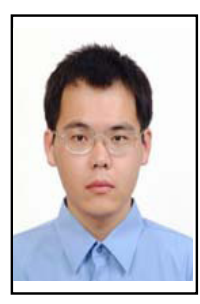

Associate Engineer. Chien-An Chen Automotive Research \& Testing Center No.6, Lugong S. $7^{\text {th }}$ Rd., Lugang, Changhua County 50544, Taiwan (R.O.C) Tel: +886-4-7811222 ext.2363

Fax: +886-4-7812336

Email:chienan@artc.org.tw

Chien-An Chen received the B.S., M.S., and Ph.D. degrees from National Yunlin University of Science and Technology, Taiwan, in 2001, 2003 and 2009, respectively. He is currently an associate Engineer in the Division of R\&D, Automotive Research \& Testing Center, Changhua, Taiwan. His research interests includes electric vehicle design, motor servo control and control theory applications.

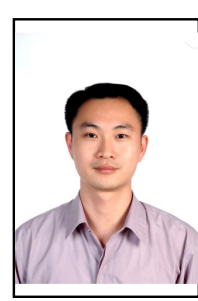

Associate Engineer. Ming-Chih Lin Automotive Research \& Testing Center No.6, Lugong S. $7^{\text {th }}$ Rd., Lugang,

Changhua County 50544, Taiwan (R.O.C)

Tel: +886-4-7811222 ext.7421

Fax: +886-4-7812336

Email: mickylin@ artc.org.tw

Ming-Chih Lin received the B.S.and M.S. degrees from Electrical Engineering of Da-Yeh University, Taiwan, in 2002 and 2005, respectively. He is currently an associate Engineer in the Division of R\&D, Automotive Research \& Testing Center, Changhua, Taiwan. His research includes electric circuit design, system control and microcontroller applications.

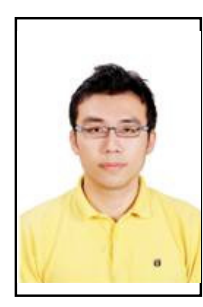

Associate Engineer. Cheng-Shun Jiang Automotive Research \& Testing Center No.6, Lugong S. $7^{\text {th }}$ Rd., Lugang, Changhua County 50544, Taiwan (R.O.C) Tel: +886-4-7811222 ext.2350

Fax: +886-4-7812336

Email: markjcs@artc.org.tw

Cheng-Shun Jiang received the B.S.and M.S. degrees from Mechanical Engineering of National Taiwan University, in 2007 and 2009, respectively. He is currently an mechanical Engineer in the Division of R\&D, Automotive Research \& Testing Center, Changhua, Taiwan. His research includes mechanism design and vehicle dynamics simulation. 